\title{
Use of Chemical Nano Selenium as Antibacterial and Antifungal Agent in Quail Diets and its Effect on Growth, Carcasses, Antioxidant, Immunity and Caecal Microbe
}

Mahmoud Alagawany ( $\nabla$ dr.mahmoud.alagwany@gmail.com )

Faculty of Agriculture, Zagazig University

\section{T. El-Saadony}

Faculty of Agriculture, Zagazig University

\section{S. S. Elnesr}

Fayoum University

\section{Farahat}

Faculty of Veterinary Medicine, Zagazig University

G. Attia

Faculty of Veterinary Medicine, Zagazig University

\section{M.A. Mahmoud}

New Valley University

M. Madkour

6Animal Production Department, National Research Centre

\section{F. M. Reda}

Faculty of Agriculture, Zagazig University

\section{Research Article}

Keywords: Nano selenium, growth, blood, pathogens, quails

Posted Date: May 12th, 2021

DOl: https://doi.org/10.21203/rs.3.rs-495839/v1

License: (9) This work is licensed under a Creative Commons Attribution 4.0 International License. Read Full License 


\section{Abstract}

Nano-minerals are used to enhance mineral bioavailability, which helps improve animal growth and health. The use of chemical nano-selenium (Che-SeNP) has lately attracted great scientific interest, mainly due to its potential benefits for poultry. The current study was conducted to investigate the impact of the dietary supplementation of Che-SeNPs on growth performance, carcass traits, blood constituents, antioxidant status, immunity, and gut microbiota of quails. A total of one week-old 180 Japanese quails were randomlydistributed into four equal groups, and each group consisted of 45 unsexed birds with five replications (nine birds each). The first group was fed a basal diet without supplementation $(0 \mathrm{~g} / \mathrm{kg}$ CheSeNP), and the second, third, and fourth groups were fed diets containing $0.2,0.4$, and $0.6 \mathrm{~g} / \mathrm{kg}$ Che-SeNP, respectively. The results showed that the dietary supplementation of Che-SeNP significantly $(P<0.05)$ increased body weight, body weight gain, and feed conversion ratio but decreased feed intake $(P<0.001)$ compared to the control group. The highest values of growth performance were recorded in the group fed $0.4 \mathrm{~g}$ Che-SeNP g/ $\mathrm{kg}$ feed. Che-SeNP levels did not affect carcass traits, relative organs (except liver), or blood hematology (except platelet count and hemoglobin level) of quails. Plasma total protein, albumin, aspartate aminotransferase (AST) and urea values were not affected by dietary Che-SeNP, but alanine aminotransferase and lactate dehydrogenase values declined. Globulin and creatinine values were linearly increased with the inclusion of Che-SeNP $(0.4$ and $0.6 \mathrm{~g} / \mathrm{kg})$ in quail diets compared to the control. The supplementation of Che-SeNP in quail diets significantly improved $(P<0.05)$ the plasma lipid profile and activities of antioxidant enzymes compared to the control group. Immunoglobulin $G$ values of Che-SeNPs $(0.4$ and $0.6 \mathrm{~g} / \mathrm{kg})$ were higher $(P<0.05)$ than those in the control group. The groups fed diets supplemented with Che-SeNP showed lower $(P<0.0001)$ total bacterial count, total yeast and molds count, Coliform, Escherichia coli, Enterobacter, and Salmonella colonization, and higher ( $P=0.0003$ and 0.0048) lactic acid bacteria counts than those in the control group. In conclusion, dietary supplementation with Che-SeNP can improve the performance, lipid profile, antioxidant indices, and immunity, as well as decrease intestinal pathogens in quails.

\section{Introduction}

Selenium (Se) is one of the elements that can be used in diets as chemical nano-selenium (Che-SeNP). Se is required for the maintenance of physiological functions, growth, and health of birds. It also plays a crucial role in nutritional value and feed metabolism, leading to considerable growth (Joshua et al., 2016). Che-SeNP has attracted more attention because of its strong adsorbing ability, high catalytic efficiency, high surface activity, and low toxicity compared to that of other chemical Se forms (Zhang et al., 2008). The high absorption of Che-SeNP from the intestinal lumen into the body was observed. Shirsat et al. (2015) highlighted that Che-SeNP has antioxidant, anticancer, antibacterial, and antiprotozoal properties. El-Deep et al. (2016) stated that dietary Che-SeNP supplementation enhanced growth performance by improving immune or antioxidative properties in broiler chicks. Additionally, Ahmadiet al. (2018) revealed that the dietary supplementation of Che-SeNP improved growth performance and immune function without deleterious effects on the internal organs. Previous 
investigations exhibited that Che-SeNP augmented body weight gain and improved antioxidant functions of broilers (Caiet al., 2012; Shirsat et al., 2015).Se nanoparticles have also been utilized in food preservation methods such as packing food items and antiseptic coating over food materials. Studies have been conducted to highlight the disinfectant properties of Se nanoparticles against

Trichophytonrubrum, Candida albicans (Kheradmandet al., 2014), Pseudomonas aeruginosa, and Proteus mirabilis (Shakibaieet al., 2015). Se can be considered an essential trace element and micronutrient for living creatures at low concentrations, but it becomes toxic and harmful at higher doses. The positive impacts of nanotechnology involving Se are well-known in many pathological conditions (Bhunchuet al., 2018). However, the inclusion of Che-SeNP in quail diet during the growth period is still limited.

It is hypothesized that the dietary addition of Che-SeNPis expected to exert beneficial effects on growing quails. Therefore, the purpose of this study was to evaluate the antibacterial and antifungal activities of Che-SeNP, and its beneficial effects on the growth, feed utilization, carcass traits, hematology, blood constituents, and cecal microbiota of growing quails.

\section{Materials And Methods}

\section{Source of selenium nanoparticles}

In this study, Che-SeNPwas prepared using wet chemicals. Sodium selenite $\left(\mathrm{Na}_{2} \mathrm{SeO}_{3}\right)$ was used for producing Se nanoparticles with ascorbic acid $\left(\mathrm{C}_{6} \mathrm{H}_{8} \mathrm{O}_{6}\right)$ as a reducing agent. A stock of aqueous solution of $100 \mathrm{mM} \mathrm{Na}_{2} \mathrm{SeO}_{3}$ and $50 \mathrm{mM} \mathrm{C}_{6} \mathrm{H}_{8} \mathrm{O}_{6}$ was prepared in a 1: 4 ratio. The solution was kept under a magnetic stirring condition at different rpm and ambient temperature for $30 \mathrm{~min}$. The mixtures were allowed to react with each other in the concentrated form until the mixture changed from colorless to red. Next, the solution was centrifuged at $3000 \mathrm{rpm}$, pellets were collected, and Che-SeNP was obtained. Chemically synthesized nano-selenium was determined via UV-Vis spectroscopy using an automated spectrometer (Spectro UV-Vis double beam UVD 3500). The morphology and element percentage of selenium nanoparticles were measured using transmission electron microscopy and an energy dispersive X-ray analytical instrument. Fourier transform infrared spectroscopy (JASCO) was used to determine the properties of produced selenium nanoparticles including size, shape, charge, and stability.

\section{Antibacterial activity of Che-SeNP}

Listeria monocytogenes ATCC 15313, Staphylococcus aureus MTCC 1809, Bacillus cereus ATCC 11778, E. coli ATCC 25922, P. aeruginosaATCC 27853, and Salmonella enterica MTCC 1253 were purchased from MIRCEN, Faculty of Agriculture, Ain Shams University (Cairo, Egypt). The antibacterial activity of CheSeNP against animal and human pathogenicgram-negative bacteria, $P$. aeruginosa ATCC 27853, E. coli ATCC 25922 , and S. enterica MTCC 1253, and gram-positive bacteria, L. monocytogenesATCC $15313, B$. cereus ATCC 11778, and S. aureus MTTC 1809,were estimated using the disc diffusion assay method. Nutrient agar medium consisting of peptone, beef extract, yeast extract, $\mathrm{NaCl}$, and agar with $5,3,5,5$, and $20 \mathrm{~g}$ in 1 liter of distilled water was prepared in slant to preserve all bacterial isolates. One hundred 
microliters of each bacterium $\left(1 \times 10^{9} \mathrm{CFU} / \mathrm{ml}\right)$ was spread with sterile swabs in Mueller-Hinton agar plates. Freshly prepared selenium nanoparticles with different concentrations $(50,100,200,400$, and 800 $\mu \mathrm{g}$ ) were loaded on paper discs (disc diameter was about $6 \mathrm{~mm}$ ) and then were placed on the Muller Hinton agar plates. Sodium selenite $(50 \mu \mathrm{g} / \mathrm{ml})$ and sterilized deionized water were loaded on paper discs and used as a positive and negative control, respectively. Mueller-Hinton agar plates were incubated for $24 \mathrm{~h}$ at $37^{\circ} \mathrm{C}$. After incubation, the obtained zones of inhibition surrounded the Che-SeNP discs were measured and recorded as the mean \pm standard deviation if they were greater than $6 \mathrm{~mm}$. The minimum inhibitory concentration (MIC) of the Che-SeNP was calculated based on a broth micro dilution method. Briefly, six pathogenic bacteria were cultured overnight at $37^{\circ} \mathrm{C}$ in Mueller-Hinton broth and were adjusted to a final density of $10^{9} \mathrm{CFU} / \mathrm{ml}$ by $0.5 \mathrm{McF}$ arland standards. The Che-SeNPs $(1 \mathrm{mg} / \mathrm{ml})$ were homogenized with sterilized deionized water and dilutions of $20-800 \mu \mathrm{g} / \mathrm{ml}$ were made. Next, $10 \mu \mathrm{l}$ of different concentrations of Che-SeNP was mixed in sterile test tubes contain $10 \mu \mathrm{l}$ of bacterial inoculum and $90 \mu$ of Mueller-Hinton broth. The test tubes were protected for a day at $37^{\circ} \mathrm{C}$. The bacterial growth was visually inspected, and the Che-SeNP with a lower level that achieved no observable bacterial growth or turbidity was considered the MIC value. The concentration of selenium nanoparticles at which complete bacterial growth was not observed was defined as the minimum bactericidal concentration. The experiments were carried out in triplicate (Reda et al., 2020).

\section{Antifungal activity of Che-SeNP}

The antifungal activity of the Che-SeNP was tested against animals and human pathogenic Candida strains.Candida albicansATCC 4862, C. glabrataATCC64677, C. parapsilosis ATCC 22019, and C. guilliermondii ATCC 6260were purchased from MIRCEN, Faculty of Agriculture, Ain Shams University (Cairo, Egypt). The antifungal activity of Che-SeNP against these four strains was evaluated viathe disc diffusion method (Hariharanet al., 2012) using sterile cotton swab lawn cultures of selected fungi that were prepared on Sabouraud Dextrose agar (SDA) plates. Che-SeNP was loaded on paper discs (disc diameter was about $6 \mathrm{~mm}$ ) and then was placed in SDA. Selenium selenite and sterilized deionized water were used as the positive and negative controls, respectively. The plates were then incubated for $36 \mathrm{~h}$ at $30^{\circ} \mathrm{C}$. The Che-SeNPs were tested for MIC using the broth dilution method (Hariharanet al., 2012). Sabouraud broth was used as diluents for fungal species. About $10^{6} \mathrm{CFU} / \mathrm{ml}$ cells could be inoculated. The Che-SeNP levels (20 to $800 \mu \mathrm{g} / \mathrm{ml}$ ) were prepared in sterilized deionized water and homogenized. Next, $10 \mu$ l of different concentrations of Che-SeNP were mixed in sterile test tubes containing $10 \mu \mathrm{l}$ of Candida inoculum and $90 \mu \mathrm{l}$ of Sabouraud broth. The test tubes were incubated at $30^{\circ} \mathrm{C}$ for $36 \mathrm{~h}$. The obtained turbidity was estimated at $600 \mathrm{~nm}$ to determine the MIC values. The least concentration of CheSeNP that reduced fungi growth by $90 \%$ was considered the MIC. The concentration of selenium nanoparticles at which complete fungal growth was not observed was defined as the minimum fungicidal concentration. The experiments were replicated in triplicate.

\section{Animals, design, and diets}

A total of 180 one week-old Japanese quails with an average weight of $27.17 \pm 0.075 \mathrm{~g}$ were used. Quail chicks were randomly allocated into four groups, and each group consisted of 45 unsexed birds with five 
replications (nine birds each).Quails were kept under traditional cages $(90 \times 40 \times 40 \mathrm{~cm})$, and feed and water were open during the study ( 4 weeks). The treatments were as follows: the first group was fed the basal ration, whereas the second, third, and fourth groups were fed diets supplemented with $0.2,0.4$, and $0.6 \mathrm{~g} / \mathrm{kg}$ of Che-SeNP, respectively. The control ration contained $24 \%$ protein, $12.53 \mathrm{MJ} / \mathrm{kg}, 0.80 \%$ calcium, $0.45 \%$ phosphorus, $1.30 \%$ lysine, and $0.92 \%$ total sulfur amino acids. Ethics statement Animal care and maintenance were conducted in accordance with the guidelines of the Egyptian Research Ethics Committee and the guidelines contained in the Guide for the Care and Use of Laboratory Animals.

\section{Growth performance and carcass measurements}

All growth parameters and feed utilization were measured at 1, 3, and 5 weeks of age. For carcass examinations, at 5 weeks old, 20 birds were randomly selected, weighed, and euthanized. All edible parts were weighed and expressed as a percent of the live body weight before euthanization.

\section{Microbiological analysis}

Ten grams of quail cecum samples (five samples per each treatment) were homogenized and transferred to a $250 \mathrm{ml}$ conical flask containing $90 \mathrm{ml}$ of sterile physiological saline solution consisting of $0.1 \%$ peptone and $0.85 \% \mathrm{NaCl}$. The mixture was well-mixed and then serially diluted up to 106 . The total bacterial count was counted using plate count agar medium at $30^{\circ} \mathrm{C}$ for $24 \mathrm{~h}$, and the total count of Enterococcus was counted using Chromocult enterococci agar medium. Total coliforms were enumerated by using MacConkey agar medium. Biochemical methods such as indole test, citrate reactions, methyl red, and Voges-Proskauer were used to identify E. coli. DeManRogosa Sharpe agar was used to enumerate the lactic acid bacteria. Salmonella Shigellaagar (SSA) media (Oxide CM 99) was used to count the Salmonella spp. The appearance of black colonies on SSA indicated the presence of Salmonella spp. SDA was used to count the molds and yeasts. SSA plates were incubated at $37^{\circ} \mathrm{C}$ for 1 day. SDA plates were incubated at $25^{\circ} \mathrm{C}$ for 3-7 days. All the obtained microbiological results were then converted to logarithmic colony-forming units per gram (CFU/g) (Reda et al., 2020).

\section{Blood chemistry}

After euthanization, blood samples were randomly collected from five quails per treatment into heparinized tubes. Hematological parameters were measured. Regarding biochemical parameters, we used a centrifuge (Janetzki, T32c, $5000 \mathrm{rpm}$, Germany) at $2146.56 \times g$ for $15 \mathrm{~min}$ to separate the plasma. The biochemical blood parameters were determined using commercial kits from Biodiagnostic Company (Giza, Egypt).

\section{Statistics}

The statistical analyses were carried out using SAS. The data of growth rate, feed efficiency, carcass parameters, hematology, blood chemistry, and microbiology were analyzed with a one-way analysis of variance. Orthogonal polynomial contrasts were used to test the significance (linear and quadratic) of the gradual levels of dietary Che-SeNP. 


\section{Results}

\section{Antibacterial activity of Che-SeNP}

Three animal and human pathogenic gram-negative bacteria (E. coli, P. aeruginosa, and S. enterica) and three gram-positive bacteria (L. monocytogenes, B. cereus, and $S$. aureus) were selected to test Che-SeNP antibacterial activity (Table 1). The maximum zones of inhibitions were observed in the three grampositive bacterial strains L. monocytogenesATCC 15313 , B. cereus ATCC 11778 , and S. aureus MTTC 1809. The antibacterial activity of Che-SeNP increased with increasing concentrations of Che-SeNP. The effect of Che-SeNP was superior to that of sodium selenite as an antimicrobial agent against tested pathogenic microorganisms;also, the deionized water did not show any antimicrobial activity. The highest MIC of Che-SeNP against E. coli ATCC 25922,P. aeruginosa ATCC 27853, and S.entericaMTCC 1253 was 45,40 , and $50 \mu \mathrm{g} / \mathrm{ml}$, respectively, whereas, the lowest MIC was 30,35 , and $25 \mu \mathrm{g} / \mathrm{ml}$ against $L$. monocytogenes ATCC 15313, S. aureus MTTC 1809, and B. cereus ATCC 11778, respectively (Table 2). 
Table 1

Zone of inhibition produced by Sodium Selenite and selenium nanoparticles

\begin{tabular}{|c|c|c|c|c|c|c|c|}
\hline \multirow[t]{2}{*}{ Item } & \multirow{2}{*}{$\begin{array}{l}\text { Sod. Selenite } \\
(50 \mu \mathrm{g} / \mathrm{mL})\end{array}$} & \multicolumn{5}{|c|}{ Selenium nanoparticles ( $\mu \mathrm{g} / \mathrm{mL})$} & \multirow{2}{*}{$\begin{array}{l}\text { DI } \\
\text { water }\end{array}$} \\
\hline & & 50 & 100 & 200 & 400 & 800 & \\
\hline Bacteria & \multicolumn{7}{|c|}{ Inhibition zones (mm) } \\
\hline $\begin{array}{l}\text { Listeria monocytogenes } \\
\text { ATCC } 15313\end{array}$ & $14 \pm 0.2 f$ & $\begin{array}{l}15 \pm \\
0.3 e\end{array}$ & $19 \pm 0.1 d$ & $\begin{array}{l}23 \pm \\
0.2 c\end{array}$ & $\begin{array}{l}26 \pm \\
0.2 b\end{array}$ & $\begin{array}{l}32 \pm \\
0.1 a\end{array}$ & - \\
\hline $\begin{array}{l}\text { Staphylococcus aureus } \\
\text { MTTC } 1809\end{array}$ & $11 \pm 0.4$ & $\begin{array}{l}13 \pm \\
0.2\end{array}$ & $18 \pm 0.2$ & $20 \pm 0.1$ & $\begin{array}{l}23 \pm \\
0.3\end{array}$ & $\begin{array}{l}28 \pm \\
0.35\end{array}$ & - \\
\hline $\begin{array}{l}\text { Bacillus cereus ATCC } \\
11778\end{array}$ & $13 \pm 0.2$ & $\begin{array}{l}16 \pm \\
0.15\end{array}$ & $20 \pm 0.1$ & $23 \pm 0.2$ & $\begin{array}{l}27 \pm \\
0.15\end{array}$ & $\begin{array}{l}33 \pm \\
0.14\end{array}$ & - \\
\hline $\begin{array}{l}\text { Escherichia coli ATCC } \\
25922\end{array}$ & $9 \pm 0.5$ & $\begin{array}{l}11 \pm \\
0.45\end{array}$ & $15 \pm 0.3$ & $17 \pm 0.4$ & $\begin{array}{l}21 \pm \\
0.2\end{array}$ & $\begin{array}{l}25 \pm \\
0.2\end{array}$ & - \\
\hline $\begin{array}{l}\text { Pseudomonas } \\
\text { aeruginosa ATCC } 27853\end{array}$ & $10 \pm 0.5$ & $\begin{array}{l}11 \pm \\
0.45\end{array}$ & $16 \pm 0.4$ & $20 \pm 0.1$ & $\begin{array}{l}22 \pm \\
0.3\end{array}$ & $\begin{array}{l}27 \pm \\
0.19\end{array}$ & - \\
\hline $\begin{array}{l}\text { Salmonella enterica } \\
\text { MTCC } 1253\end{array}$ & $8 \pm 0.5 f$ & $\begin{array}{l}11 \pm \\
0.45\end{array}$ & $14 \pm 0.5$ & $17 \pm 0.4$ & $\begin{array}{l}20 \pm \\
0.5\end{array}$ & $\begin{array}{l}24 \pm \\
0.3 a\end{array}$ & - \\
\hline \multicolumn{8}{|l|}{ Fungi } \\
\hline $\begin{array}{l}\text { Candida albicans ATCC } \\
4862\end{array}$ & $11 \pm 0.4 f$ & $\begin{array}{l}12 \pm \\
0.3\end{array}$ & $14 \pm 0.2$ & $\begin{array}{l}15 \\
\pm \\
0.2\end{array}$ & $\begin{array}{l}16 \pm \\
0.15\end{array}$ & $\begin{array}{l}17 \pm \\
0.1 \mathrm{a}\end{array}$ & - \\
\hline $\begin{array}{l}\text { Candida glabrata ATCC } \\
64677\end{array}$ & $8 \pm 0.5 \mathrm{e}$ & $\begin{array}{l}9 \pm \\
0.5 \mathrm{~d}\end{array}$ & $9 \pm 0.5 d$ & $\begin{array}{l}10 \\
\pm \\
0.4 \mathrm{c}\end{array}$ & $\begin{array}{l}11 \pm \\
0.4 \mathrm{~b}\end{array}$ & $\begin{array}{l}13 \pm \\
0.2 \mathrm{a}\end{array}$ & - \\
\hline $\begin{array}{l}\text { Candida parapsilosis } \\
\text { ATCC } 22019\end{array}$ & $10 \pm 0.35 d$ & $\begin{array}{l}11 \pm \\
0.4 \mathrm{c}\end{array}$ & $12 \pm 0.3 b$ & $\begin{array}{l}13 \\
\pm \\
0.3 b\end{array}$ & $\begin{array}{l}14 \pm \\
0.2 \mathrm{a}\end{array}$ & $\begin{array}{l}14 \pm \\
0.3 a\end{array}$ & - \\
\hline $\begin{array}{l}\text { Candida guilliermondii } \\
\text { ATCC } 6260\end{array}$ & $8 \pm 0.5 d$ & $\begin{array}{l}8 \pm \\
0.5 \mathrm{~d}\end{array}$ & $9 \pm 0.5 c$ & $\begin{array}{l}9 \pm \\
0.5 \mathrm{c}\end{array}$ & $\begin{array}{l}10 \pm \\
0.3 \mathrm{~b}\end{array}$ & $\begin{array}{l}11 \pm \\
0.5 a\end{array}$ & - \\
\hline \multicolumn{8}{|l|}{ Mean \pm SE } \\
\hline Mean in the same raw wi & erent small & $\mathrm{sa}$ & ificantly & erent & 05 & & \\
\hline
\end{tabular}


Table 2

The MIC (Minimum Inhibitory Concentration), MBC (Minimum

Bactericidal Concentration) and MFC (Minimum fungicidal concentration) of the selenium nanoparticles.

\begin{tabular}{|lll|}
\hline Microorganisms & \multicolumn{2}{l|}{ Selenium nanoparticles } \\
\cline { 2 - 3 } & MIC $\mu \mathrm{g} / \mathrm{mL}$ & MBC $\mu \mathrm{g} / \mathrm{mL}$ \\
\hline Bacteria & & \\
\hline Listeria monocytogenes ATCC 15313 & 30 & 60 \\
\hline Staphylococcus aureus MTTC 1809 & 35 & 70 \\
\hline Bacillus cereus ATCC 11778 & 25 & 50 \\
\hline Escherichia coli ATCC 25922 & 45 & 90 \\
\hline Pseudomonas aeruginosa ATCC 27853 & 40 & 80 \\
\hline Salmonella enterica MTCC 1253 & 50 & 100 \\
\hline Fungi & & \\
\hline Candida albicans ATCC 4862 & 70 & 140 \\
\hline Candida glabrata ATCC 64677 & 90 & 180 \\
\hline Candida parapsilosis ATCC 22019 & 80 & 160 \\
\hline Candida guilliermondii ATCC 6260 & 100 & 200 \\
\hline
\end{tabular}

\section{Antifungal activity of Che-SeNP}

Che-SeNP showed acceptable antifungal activity, ranging from $50 \mu \mathrm{g} / \mathrm{ml}$ to $800 \mu \mathrm{g} / \mathrm{ml}$, against all the tested fungal strains. $C$. albicans ATCC 4862 was the most sensitive strainto Che-SeNP when compared to other tested strains. The MICs for $C$. albicans ATCC 4862, C. glabrataATCC64677, C. parapsilosis ATCC 22019, and C. guilliermondii ATCC 6260 were 70, 90, 80, and $100 \mu \mathrm{g} / \mathrm{ml}$, respectively (Table 2).

\section{Growth performance}

The effects of dietary Che-SeNP supplementation on the growth performance of Japanese quails are presented in Table 3. From the results, quails fed diets containing Che-SeNP had significantly higher (linear and quadratic, $\mathrm{P}<0.05)$ body weight $(\mathrm{BW})$ and body weight gain $(\mathrm{BWG})$ during the experimental periods. The group fed diets containing Che-SeNP $(0.4 \mathrm{~g} / \mathrm{kg}$ diet $)$ had the highest BW and BWG. Feed intake was decreased (linear and quadratic, $\mathrm{P}<0.001$ ) in the Che-SeNP groups compared with that in the control group. The feed conversion ratio was linearly and quadratically improved with the addition of CheSeNP in quail diets during all periods. Generally, the best growth performance parameters were recorded in the group fed $0.4 \mathrm{~g}$ Che-SeNP/ $\mathrm{kg}$ feed. 
Table 3

Growth performance of growing Japanese quail as affected by dietary treatments

\begin{tabular}{|c|c|c|c|c|c|c|c|}
\hline \multirow[t]{2}{*}{ Items } & \multicolumn{4}{|c|}{ Chemical nano selenium levels (g/kg diet) } & \multirow[t]{2}{*}{ SEM } & \multicolumn{2}{|l|}{$P$ value } \\
\hline & 0 & 0.2 & 0.4 & 0.6 & & Linear & Quadratic \\
\hline \multicolumn{8}{|c|}{ Body weight (g) } \\
\hline $1 \mathrm{wk}$ & 27.14 & 27.16 & 27.19 & 27.15 & 0.049 & 0.8122 & 0.5978 \\
\hline $3 \mathrm{wk}$ & $92.58^{b}$ & $98.40^{\mathrm{a}}$ & $97.52^{\mathrm{a}}$ & $97.77^{a}$ & 0.717 & 0.0024 & 0.0060 \\
\hline $5 w k$ & $178.51^{c}$ & $190.75^{b}$ & $198.07^{a}$ & $193.68^{a b}$ & 1.354 & $<.0001$ & 0.0004 \\
\hline \multicolumn{8}{|c|}{$\begin{array}{l}\text { Body weight gain (g / } \\
\text { day) }\end{array}$} \\
\hline $1-3 w k$ & $4.67^{b}$ & $5.09^{a}$ & $5.02^{a}$ & $5.04^{\mathrm{a}}$ & 0.051 & 0.0026 & 0.0064 \\
\hline $3-5 w k$ & $6.14^{c}$ & $6.60^{b}$ & $7.18^{a}$ & $6.85^{\mathrm{ab}}$ & 0.101 & 0.0007 & 0.0081 \\
\hline $1-5 w k$ & $5.41^{\mathrm{C}}$ & $5.84^{\mathrm{b}}$ & $6.10^{\mathrm{a}}$ & $5.95^{\mathrm{ab}}$ & 0.051 & $<.0001$ & 0.0005 \\
\hline \multicolumn{8}{|c|}{ Feed intake (g / day) } \\
\hline $1-3 w k$ & $14.06^{\mathrm{a}}$ & $12.89^{\mathrm{c}}$ & $13.22^{b}$ & $13.14^{\mathrm{bc}}$ & 0.079 & 0.0002 & 0.0002 \\
\hline $3-5 w k$ & $23.71^{\mathrm{a}}$ & $19.91^{\mathrm{c}}$ & $21.02^{b}$ & $20.22^{\mathrm{c}}$ & 0.202 & $<.0001$ & 0.0001 \\
\hline $1-5 w k$ & $18.89^{a}$ & $16.4^{\mathrm{c}}$ & $17.12^{b}$ & $16.68^{c}$ & 0.083 & $<.0001$ & $<.0001$ \\
\hline \multicolumn{8}{|c|}{$\begin{array}{l}\text { Feed conversion ratio } \\
(\mathrm{g} / \mathrm{g})\end{array}$} \\
\hline $1-3 w k$ & $3.01^{a}$ & $2.53^{b}$ & $2.63^{b}$ & $2.60^{\mathrm{b}}$ & 0.038 & 0.0002 & 0.0005 \\
\hline $3-5 w k$ & $3.86^{a}$ & $3.02^{b}$ & $2.93^{c}$ & $2.95^{\mathrm{bc}}$ & 0.018 & $<.0001$ & $<.0001$ \\
\hline $1-5 w k$ & $3.49^{\mathrm{a}}$ & $2.81^{b}$ & $2.81^{\mathrm{b}}$ & $2.80^{\mathrm{b}}$ & 0.015 & $<.0001$ & $<.0001$ \\
\hline
\end{tabular}

\section{Carcass traits}

As indicated in Table 4, dietary Che-SeNP levels did not affect carcass traits and relative organs (except liver) of Japanese quails. Supplementation of Che-SeNP significantly increased the relative weight of the liver (linear and quadratic, $\mathrm{P}<0.05$ ) compared to the control group. 
Table 4

Carcass traits and relative organs of growing Japanese quail as affected by dietary treatments.

\begin{tabular}{|c|c|c|c|c|c|c|c|}
\hline \multirow[t]{2}{*}{ Items } & \multicolumn{4}{|c|}{ Chemical nano selenium levels ( $\mathrm{g} / \mathrm{kg}$ diet) } & \multirow[t]{2}{*}{ SEM } & \multicolumn{2}{|l|}{$P$ value } \\
\hline & 0 & 0.2 & 0.4 & 0.6 & & Linear & Quadratic \\
\hline Carcass \% & 73.67 & 76.10 & 71.72 & 72.82 & 1.087 & 0.1988 & 0.5658 \\
\hline Liver \% & $2.22^{\mathrm{b}}$ & $2.84^{\mathrm{a}}$ & $2.85^{\mathrm{a}}$ & $2.69^{a}$ & 0.099 & 0.0355 & 0.0144 \\
\hline Gizzard \% & 2.54 & 2.30 & 2.10 & 2.35 & 0.208 & 0.5312 & 0.3718 \\
\hline Heart \% & 1.01 & 1.14 & 1.03 & 0.94 & 0.076 & 0.4728 & 0.2670 \\
\hline Giblets \% & 5.76 & 6.28 & 5.98 & 5.98 & 0.344 & 0.8382 & 0.5059 \\
\hline Dressing \% & 79.44 & 82.39 & 77.70 & 78.79 & 1.279 & 0.3004 & 0.5093 \\
\hline
\end{tabular}

\section{Blood hematology}

The effects of the addition of Che-SeNP on the blood hematology of growing quails are presented in Table 5. Dietary Che-SeNP levels did not affect $(P>0.05)$ white blood cells (WBCs), lymphocytes, midrange, granulocytes, red blood cells (RBCs), hematocrit, and mean corpuscular volume of the growing quails. Platelet count and hemoglobin $(\mathrm{Hb})$ levels were increased (linear, $\mathrm{P}<0.05$ ) by the addition of CheSeNP at 0.4 and $0.6 \mathrm{~g} / \mathrm{kg}$. Mean corpuscular volume value was augmented (linear, $P<0.05$ ) by the addition of Che-SeNP level compared to the control group (without Che-SeNP). The supplementation of dietary Che-SeNP at levels of $0.6 \mathrm{~g} / \mathrm{kg}$ decreased values of red blood cell distribution width linearly $(P=$ 0.0091 ), whereas the dietary level 0.4 and $0.6 \mathrm{~g} / \mathrm{kg}$ declined values of red blood cell distribution volume linearly $(P=0.0019)$ compared to the control group. 
Table 5

Hematological parameters of growing Japanese quail as affected by dietary treatments.

\begin{tabular}{|c|c|c|c|c|c|c|c|}
\hline \multirow[t]{2}{*}{ Items ${ }^{1}$} & \multicolumn{4}{|c|}{ Chemical nano selenium levels ( $\mathrm{g} / \mathrm{kg}$ diet) } & \multirow[t]{2}{*}{ SEM } & \multicolumn{2}{|l|}{$P$ value } \\
\hline & 0 & 0.2 & 0.4 & 0.6 & & Linear & Quadratic \\
\hline $\begin{array}{l}\text { WBCs }\left(10^{3} /\right. \\
\mu l)\end{array}$ & 22.43 & 23.32 & 23.73 & 23.67 & 1.972 & 0.5754 & 0.7496 \\
\hline LYM (\%) & 93.65 & 93.15 & 93.14 & 94.61 & 0.629 & 0.3485 & 0.1632 \\
\hline MID (\%) & 5.84 & 6.41 & 6.50 & 3.14 & 0.771 & 0.0594 & 0.0422 \\
\hline GRA (\%) & 0.32 & 0.44 & 0.36 & 0.17 & 0.084 & 0.2690 & 0.1746 \\
\hline $\begin{array}{l}\mathrm{RBCs}\left(10^{6} /\right. \\
\mu \mathrm{ll})\end{array}$ & 2.34 & 2.60 & 2.63 & 2.75 & 0.196 & 0.1943 & 0.7486 \\
\hline HGB (g/dL) & $9.27^{\mathrm{b}}$ & $11.77^{\mathrm{ab}}$ & $12.40^{\mathrm{a}}$ & $13.37^{a}$ & 0.822 & 0.0099 & 0.3988 \\
\hline HCT (\%) & 32.30 & 35.83 & 29.40 & 21.20 & 3.438 & 0.0327 & 0.1271 \\
\hline $\operatorname{MCV}\left(\mu m^{3}\right)$ & 137.83 & 137.90 & 127.10 & 124.90 & 2.985 & 0.0143 & 0.7582 \\
\hline $\mathrm{MCH}(\mathrm{pg})$ & $40.87^{c}$ & $47.90^{\mathrm{b}}$ & $53.87^{a}$ & $56.93^{a}$ & 1.167 & $<.0001$ & 0.1626 \\
\hline RDWSD & $51.87^{a}$ & $52.37^{a}$ & $44.00^{\mathrm{ab}}$ & $41.47^{b}$ & 2.420 & 0.0091 & 0.5743 \\
\hline RDWCV & $13.07^{a}$ & $13.30^{\mathrm{a}}$ & $11.87^{b}$ & $11.17^{\mathrm{b}}$ & 0.349 & 0.0019 & 0.2220 \\
\hline $\begin{array}{l}\mathrm{PLT}\left(10^{3} /\right. \\
\mu \mathrm{ll})\end{array}$ & $5.67^{b}$ & $8.67^{b}$ & $16.67^{a}$ & $15.00^{\mathrm{a}}$ & 2.771 & 0.0327 & 0.4763 \\
\hline \multicolumn{8}{|c|}{$\begin{array}{l}\text { Means in the same raw with no superscript letters after them or with a common superscript letter } \\
\text { following them are not significantly different }(P<0.05) \text {. }\end{array}$} \\
\hline \multicolumn{8}{|c|}{$\begin{array}{l}{ }^{1} \text { WBCs: white blood cells; LYM: lymphocytes; MID: mid-range; GRA: granulocytes; RBCs: red blood } \\
\text { cells; HGB: hemoglobin; HCT: hematocrit; MCV: Mean corpuscular volume; MCH: Mean corpuscular } \\
\text { hemoglobin; RDWSD: Red blood cell distribution width; RDWCV: Red blood cell distribution volume; } \\
\text { PLT: Platelet count; }\end{array}$} \\
\hline
\end{tabular}

\section{Blood constituents}

Liver and kidney function data are presented in Table 6. The total protein and albumin were not affected $(P>0.05)$ by dietary Che-SeNP. The globulin value was lowest (linear, $P<0.05)$ in the Che-SeNP levels of 0.4 and $0.6 \mathrm{~g} / \mathrm{kg}$ compared with that in the Che-SeNP levels of $0.2 \mathrm{~g} / \mathrm{kg}$ and the control group. The quails fed diets containing Che-SeNP had higher alanine aminotransferase (ALT) and lactate dehydrogenase (P $<0.05$ ) than those in the control group. Dietary Che-SeNP had no significant effect on AST and urea values. The inclusion of Che-SeNP $(0.4$ and $0.6 \mathrm{~g} / \mathrm{kg})$ in quail diets increased the creatinine value (linear, $\mathrm{P}<0.05)$ compared with that in the control and $0.2 \mathrm{~g} / \mathrm{kg}$ Che-SeNP groups. 
Table 6

Liver and kidney function of growing Japanese quail as affected by dietary treatments.

\begin{tabular}{|c|c|c|c|c|c|c|c|}
\hline \multirow[t]{2}{*}{ Items ${ }^{1}$} & \multicolumn{4}{|c|}{ Chemical nano selenium levels ( $\mathrm{g} / \mathrm{kg}$ diet) } & \multirow[t]{2}{*}{ SEM } & \multicolumn{2}{|l|}{$P$ value } \\
\hline & 0 & 0.2 & 0.4 & 0.6 & & Linear & Quadratic \\
\hline $\mathrm{TP}(\mathrm{g} / \mathrm{dL})$ & 3.27 & 2.88 & 3.22 & 3.35 & 0.109 & 0.3323 & 0.0670 \\
\hline ALB (g/dL) & 1.20 & 1.21 & 1.27 & 1.16 & 0.026 & 0.7073 & 0.0859 \\
\hline GLOB $(\mathrm{g} / \mathrm{dL})$ & $1.63^{\mathrm{c}}$ & $1.72^{\mathrm{c}}$ & $1.95^{\mathrm{b}}$ & $2.19^{\mathrm{a}}$ & 0.066 & 0.0003 & 0.3285 \\
\hline A/G (\%) & $0.74^{\mathrm{a}}$ & $0.71^{\mathrm{a}}$ & $0.65^{\mathrm{a}}$ & $0.53^{b}$ & 0.022 & 0.0005 & 0.1222 \\
\hline AST (IU/L) & 221.15 & 229 & 237.75 & 238.3 & 4.207 & 0.0305 & 0.5003 \\
\hline ALT (IU/L) & $10.82^{c}$ & $12.87^{b}$ & $13.72^{b}$ & $16.67^{a}$ & 0.493 & $<.0001$ & 0.4149 \\
\hline LDH (IU/L) & $119.45^{c}$ & $143.5^{a}$ & $133.20^{b}$ & $143.95^{\mathrm{a}}$ & 1.328 & $<.0001$ & 0.0001 \\
\hline Creatinine (mg/dL) & $0.33^{b}$ & $0.33^{b}$ & $0.42^{\mathrm{a}}$ & $0.44^{\mathrm{a}}$ & 0.017 & 0.0008 & 0.6602 \\
\hline Urea $(\mathrm{mg} / \mathrm{dL})$ & 6.86 & 7.03 & 7.12 & 7.24 & 0.098 & 0.0503 & 0.8386 \\
\hline \multicolumn{8}{|c|}{$\begin{array}{l}\text { Means in the same raw with no superscript letters after them or with a common superscript letter } \\
\text { following them are not significantly different }(P<0.05) \text {. }\end{array}$} \\
\hline
\end{tabular}

The effects of Che-SeNP inclusion in diets on the lipid profile of quails are presented in Table 7. Total cholesterol, triglyceride, and very-low-density lipoprotein were significantly decreased $(P<0.05)$ in CheSeNP-treated groups compared to those in the control. The dietary supplementation of Che-SeNP $(0.2$ and $0.4 \mathrm{~g} / \mathrm{kg})$ quadratically increased high-density lipoprotein $(\mathrm{HDL})(P=0.0019)$. 
Table 7

Lipid profile of growing Japanese quail as affected by dietary treatments.

\begin{tabular}{|c|c|c|c|c|c|c|c|}
\hline \multirow[t]{2}{*}{ Items $^{1}$} & \multicolumn{4}{|c|}{ Chemical nano selenium levels ( $\mathrm{g} / \mathrm{kg}$ diet) } & \multirow[t]{2}{*}{ SEM } & \multicolumn{2}{|l|}{$P$ value } \\
\hline & 0 & 0.2 & 0.4 & 0.6 & & Linear & Quadratic \\
\hline $\mathrm{TC}(\mathrm{mg} / \mathrm{dL})$ & $153.55^{a}$ & $143.40^{\mathrm{b}}$ & $144.50^{\mathrm{ab}}$ & $125.25^{c}$ & 1.912 & 0.0002 & 0.1585 \\
\hline $\mathrm{TG}(\mathrm{mg} / \mathrm{dL})$ & $298.75^{a}$ & $225.00^{b}$ & $210.00^{b c}$ & $192.00^{c}$ & 5.882 & $<.0001$ & 0.0019 \\
\hline $\mathrm{HDL}(\mathrm{mg} / \mathrm{dL})$ & $35.30^{c}$ & $46.08^{b}$ & $56.83^{\mathrm{a}}$ & $38.92^{\mathrm{bc}}$ & 3.092 & 0.1640 & 0.0019 \\
\hline $\mathrm{LDL}(\mathrm{mg} / \mathrm{dL})$ & $58.51^{\mathrm{a}}$ & $52.28^{b}$ & $45.68^{c}$ & $47.94^{c}$ & 2.911 & 0.0236 & 0.2043 \\
\hline $\operatorname{VLDL}(\mathrm{mg} / \mathrm{dL})$ & $59.75^{\mathrm{a}}$ & $45.00^{\mathrm{b}}$ & $42.00^{\mathrm{bc}}$ & $38.40^{\mathrm{C}}$ & 1.176 & $<.0001$ & 0.0019 \\
\hline $\mathrm{SOD}(\mathrm{U} / \mathrm{ml})$ & $0.12^{\mathrm{c}}$ & $0.22^{\mathrm{b}}$ & $0.29^{\mathrm{a}}$ & $0.22^{b}$ & 0.004 & $<.0001$ & $<.0001$ \\
\hline $\mathrm{MDA}(\mathrm{nmol} / \mathrm{mL})$ & $0.33^{a}$ & $0.24^{b}$ & $0.22^{b}$ & $0.13^{c}$ & 0.007 & $<.0001$ & 0.7918 \\
\hline $\mathrm{GSH}(\mathrm{ng} / \mathrm{ml})$ & $0.11^{c}$ & $0.22^{b}$ & $0.28^{a}$ & $0.26^{\mathrm{a}}$ & 0.008 & $<.0001$ & 0.0001 \\
\hline $\operatorname{GPX}(\mathrm{mg} / \mathrm{dl})$ & $0.13^{d}$ & $0.23^{c}$ & $0.30^{b}$ & $0.34^{a}$ & 0.007 & $<.0001$ & 0.0058 \\
\hline $\operatorname{lgG}(\mathrm{mg} / \mathrm{dl})$ & $0.89^{b}$ & $1.33^{a}$ & $1.19^{a}$ & $1.17^{a}$ & 0.042 & 0.0127 & 0.0021 \\
\hline $\operatorname{lgM}(\mathrm{mg} / \mathrm{dl})$ & $0.49^{c}$ & $0.56^{\mathrm{bc}}$ & $0.64^{b}$ & $0.90^{\mathrm{a}}$ & 0.026 & $<.0001$ & 0.0238 \\
\hline $\lg A(m g / d l)$ & $0.53^{b}$ & $0.64^{\mathrm{b}}$ & 0.80 & $0.85^{a}$ & 0.034 & 0.0007 & 0.5469 \\
\hline Selenium & $0.07^{c}$ & $0.19^{b}$ & $0.24^{\mathrm{ab}}$ & $0.27^{a}$ & 0.016 & 0.0001 & 0.0604 \\
\hline \multicolumn{8}{|c|}{$\begin{array}{l}\text { Means in the same raw with no superscript letters after them or with a common superscript letter } \\
\text { following them are not significantly different }(P<0.05) \text {. }\end{array}$} \\
\hline \multicolumn{8}{|c|}{${ }^{1}$ TC: total cholesterol; TG: triglycerides; HDL: high density lipoprotein; LDL: low density lipoprotein. } \\
\hline
\end{tabular}

\section{Antioxidant indices}

The results of the antioxidant indices in the serum are given in Table 7. The activities of superoxide dismutase (SOD) and glutathione peroxidase, and the levels of reduced glutathione (GSH) were significantly increased (linear and quadratic, $\mathrm{P}<0.05$ ) by the dietary supplementation of Che-SeNP compared with those in the control. Dietary Che-SeNP levels decreased malondialdehyde (MDA) levels linearly $(P<0.0001)$ compared to the control group. The values of immunoglobulin $G(\operatorname{lgG})$ of Che-SeNPtreated groups were higher (linear and quadratic, $\mathrm{P}<0.05$ ) than those in the control group. IgM and IgA values of Che-SeNP $(0.4$ and $0.6 \mathrm{~g} / \mathrm{kg})$ were higher $(P<0.05)$ than those in the control group. The quails 
fed a diet supplemented with Che-SeNP showed higher plasma selenium concentrations when compared to those fed the control diet (linear, $\mathrm{P}=0.0001$ ).

\section{Microbiological analysis}

The different Che-SeNPlevels significantly affected the cecal microbiota of growing Japanese quails (Table 8). The groups fed a diet supplemented with Che-SeNP showed lower total bacterial count, total yeast and molds count, Coliform, E. coli, Enterobacter, and Salmonella colonization than those in the control group (linear and quadratic, $\mathrm{P}<0.0001$ ). However, the dietary supplementation of Che-SeNP levels increased the lactic acid bacteria count (linear and quadratic, $\mathrm{P}<0.05$ ) compared to the control group.

Table 8

Caecal microbiota of growing Japanese quail as affected by dietary treatments

\begin{tabular}{|c|c|c|c|c|c|c|c|}
\hline \multirow[t]{2}{*}{ Items } & \multicolumn{4}{|c|}{$\begin{array}{l}\text { Chemical nano selenium levels }(\mathrm{g} / \mathrm{kg} \\
\text { diet) }\end{array}$} & \multirow[t]{2}{*}{ SEM } & \multicolumn{2}{|l|}{$P$ value } \\
\hline & 0 & 0.2 & 0.4 & 0.6 & & Linear & Quadratic \\
\hline \multicolumn{8}{|c|}{$\begin{array}{l}\text { Microbiological count (Log } \\
\text { CFU/g) }\end{array}$} \\
\hline TBC & $6.05^{\mathrm{a}}$ & $5.32^{\mathrm{b}}$ & $5.08^{c}$ & $5.31^{b}$ & 0.006 & $<.0001$ & $<.0001$ \\
\hline TYMC & $5.82^{\mathrm{a}}$ & $5.20^{\mathrm{b}}$ & $4.90^{d}$ & $5.12^{c}$ & 0.014 & $<.0001$ & $<.0001$ \\
\hline Coliform & $5.96^{\mathrm{a}}$ & $5.26^{\mathrm{b}}$ & $5.08^{\mathrm{c}}$ & $4.92^{d}$ & 0.035 & $<.0001$ & 0.0004 \\
\hline E. coli & $5.93^{\mathrm{a}}$ & $5.25^{\mathrm{b}}$ & $5.05^{c}$ & $5.09^{c}$ & 0.022 & $<.0001$ & $<.0001$ \\
\hline Lactic acid bacteria & $5.31^{\mathrm{c}}$ & $5.58^{a}$ & $5.40^{\mathrm{b}}$ & $5.53^{\mathrm{a}}$ & 0.017 & 0.0003 & 0.0048 \\
\hline Enterobacter & $5.86^{\mathrm{a}}$ & $5.23^{\mathrm{b}}$ & $4.97^{c}$ & $5.08^{c}$ & 0.026 & $<.0001$ & $<.0001$ \\
\hline Salmonella & $6.38^{a}$ & $3.22^{b}$ & $2.23^{c}$ & $2.07^{c}$ & 0.057 & $<.0001$ & $<.0001$ \\
\hline
\end{tabular}

\section{Discussion}

Antimicrobial agents are critical in the pharmaceutical and textile industries, water purification, and food packaging. One notable disadvantage of organically synthesized compounds is toxicity in the body; therefore, the trend is to use inorganic nanoparticles such as Che-SeNPs that have antimicrobial activity (Phull et al., 2016). These Che-SeNPs have an inhibitory effect on many microorganisms such as bacteria, fungi, and nematodes. Currently, antimicrobial drugs are becoming less effective for many diseases globally because of the drug resistance capability of microbes. Microorganisms use their biofilm to resist antimicrobial drugs, and the membranes are the primary source of food contamination. Che-SeNPs have been used to control the growth and formation of biofilms of food spoilage bacteria, 
including B. cereus, Enterococcus faecalis, S. aureus, E. coli 0157:H7, S. typhimurium, and S. enterica(Khiralla and El-deeb, 2015). Developing more effective antibacterial agents is vital for a wide range of applications in various diseases for better public health. However, the emergence of multiple antibiotic-resistant bacteria presents a public health threat. Many developed antimicrobial drugs have limited effective applications due to chemical imbalance, low biocompatibility, and poor long-term antibacterial efficiency. Che-SeNPs conjugated with quercetin and acetylcholine have shown a great antimicrobial effect on the pathogen (Huang et al., 2016). Probiotics are microorganisms that can improve intestinal microbial balance and provide benefits to human health after consumption in adequate amounts. Lactobacillus plantarumand L. johnsoniicells are resistant against selenium dioxide, and their cell-free extracts were tested against C. albicansATCC 14053 (Kheradmandet al., 2014). Selenium particles extracted from cultures of $S$. carnosusstabilized by their natural protein coating, for instance, show considerable activity against the nematode Steinernemafeltiae, Saccharomyces cerevisiae, and E. coli. Natural SeNPs were found more active than mechanically generated selenium particles and can be applied as antimicrobial materials in medicine and agriculture (Estevamet al., 2017). Antimicrobial tests show SeNP activity against S.epidermidisbut not against $E$. coli in a low Se concentration of $2 \mathrm{ppm}$. S. aureusis an important bacterium commonly found in numerous infections. $S$. aureusinfections were difficult to treat due to their biofilm formation and defined antibiotic resistance. SeNPs were used effectively in the prevention and treatment of disease caused by $S$. aureus(Reda et al., 2020).

The antifungal activity of SeNPs was evaluated against C. albicans ATCC 4862, C. glabrataATCC64677, C. parapsilosis ATCC 22019, and C. guilliermondii ATCC 6260 usingthe disk diffusion method (Hariharanet al., 2012) (Table 1). The common antifungal agents are enormously irritant and lethal, and it is necessary to formulate newer types of safe and cost-effective fungicidals. Accordingly, the results of the present study illustrate that selenium nanoparticles have good antifungal activity against all pathogenic animals and human Candida species. Selenium nanoparticlesshowed better activity against C. albicansATCC 4862 compared to other Candidaspecies used in this study. In addition, it was proved that SeNPs have low toxicity and high biological activities (Fesharakiet al., 2010). A similar observation was reported byShakibaie etal. (2015), who studied the antifungal activity of selenium nanoparticles against Aspergillus fumigatus and $C$. albicans,and found theMICs for A.fumigatus and $C$. albicansto be 100 and $70 \mu \mathrm{g} / \mathrm{mL}$, respectively. However,the high surface-to-volume ratios and their nanoscale sizes provide better activity against biological materials. In addition, Che-SeNP has a significantly lower toxicity than other inorganic and organic forms of supplemental selenium (Shakibaie et al., 2015).

The current data demonstrated that dietary supplementation with Che-SeNP substantially affected BW, BWG, feed intake, and feed conversion ratio (FCR). A similar observation was stated by Zhou and Wang (2011), who clarified significant improvement in the FCR and growth performance by supplementation with Che-SeNP up to a $0.5 \mathrm{mg} / \mathrm{kg}$ basal diet. Khazraie and Ghazanfarpoor (2015) illustrated that weight gain was significantly increased in quail chicks fed the Che-SeNP-supplemented diet compared to the control. Selim et al. (2015), using the Che-SeNP form (0.15 to $0.30 \mathrm{ppm})$, showed a marked improvement in BW, BWG, and FCR of broiler chicks. Ibrahim et al. (2020) indicated that dietary Che-SeNP 
supplementation significantly improved BW, BWG, and FCR of broiler chicks compared to the control group. The improved performance may be due to (1) higher utilization of Che-SeNP associated with the unique properties of nano form, such as excellent bioavailability higher solubility, high cellular uptake, and greater surface activity (Zhang et al., 2008); (2) the involvement of Se in regulating several enzymatic systems, which interferes in energy metabolism and metabolism of the essential fatty acid apurinic and apyrimidinic base; and (3) Che-SeNP has high biological activity, immune regulation, and oxidation resistance (Huang et al., 2016). In addition, the improved FCR can be elucidated by the Che-SeNP role in enhancing the activity of intestinal microbiota to digest and absorb the nutrients via the intestinal barriers (Yoon et al., 2007).

The results of the present study in carcass traits and relative organ weight of growing Japanese quails were in line with the study of Khazraie and Ghazanfarpoor (2015), who stated that the supplementation of Che-SeNP to the diet did not affect carcass traits of chicks. Additionally, Caiet al. (2012) reported no significant effect of Che-SeNP on the weights of carcass parts in broilers. Selim et al. (2015) indicated that giblets were not affected due to the inclusion of Che-SeNP in the diet. Recently, Bakhshalinejadet al. (2019) reported that neither carcass yield nor carcass yield parts such as thigh and breast muscles, as well as liver, gizzard, and heart of broilers, was affected by different Che-SeNP levels at $42 \mathrm{~d}$ of age.

Boostaniet al. (2015) exhibited that packed cell volume, RBCs and WBCs were not different between the birds supplemented with Che-SeNP and the control birds, which is in line with the results of the current study. Likewise, Chen et al. (2013) revealed no significant difference in WBCs, RBCs and packed cell volume of broilers fed different Se sources. Additionally, Mohamed et al. (2016) illustrated that using CheSeNP in the diet of Sinai chicks did not significantly affect WBCs, eosinophils, and monocytes. However, our study stated that $\mathrm{Hb}$ level was increased by the addition of Che-SeNP, in agreement with Khazraie and Ghazanfarpoor (2015), who reported a significant increase in $\mathrm{Hb}$ concentration in quails fed a diet containing Che-SeNP. These findings may be caused by Se enhancing the activity of hemopoietic organs (Tayeb and Qader, 2012).

The results of the current study on the blood biochemistry of quails were in agreement with previous studies. Serum total protein and albumin were not significantly affected due to Che-SeNP supplementation to the broiler diet (Selim et al., 2015). Serum globulin levels were increased with the addition of Che-SeNP in the diet (Mohamed et al., 2016). Additionally, no significant difference in serum AST activity was observed of chicks fed a diet supplemented with Che-SeNP (Selimet al., 2015). However, our results are similar to the study of Elsaid (2015), who reported increased serum ALT activity in birds fed a diet supplemented with Che-SeNP. Selim et al. (2015) found that increasing the Che-SeNP level in broiler diets led to an increase in plasma creatinine levels compared to the control group. However, some studies showed that blood creatinine levels declined in birds fed a diet containing Che-SeNP (Elsaid, 2015). The potential reason for these differences is possibly related to the dose and time of animal exposure. We concluded from the current study that the higher Che-SeNP level is the cause of increased ALT and creatinine as indicators of liver and kidney oxidative damage, whereas lower levels showed less damage. 
Selenium has a hypocholesterolemic activity. A significant reduction in plasma TC and an increase in $\mathrm{HDL}$ were detected in the Che-SeNP-treated birds. The dietary addition of nan forms of selenium for hens caused significant declines in serum levels of cholesterol as compared to that of the control (Ismail et al., 2016). Rizk et al. (2017) stated that Che-SeNP addition in the chicken diet decreased cholesterol, triglycerides, and low-density lipoproteins as well as increased HDL compared with the control group. These results might be attributed to lipolysis that increased with Se intake. Additionally, the reduction of cholesterol may be due to the role of Se in the activation of peroxisome proliferator-activated receptor- $\gamma$ that can decrease sterol regulatory element-binding protein-2 level, resulting in decreased cholesterol synthesis (Klopotek et al., 2006).

The antioxidant system is greatly influenced by the nutritional status of an animal. Se nanoparticles have vital roles in protecting the body cells from reactive oxygen species abundance by decreasing the production of free radicals and lipid peroxidation (Pilarczyket al., 2012). Se is well-known for its ability to boost the antioxidant capacity as it forms selenocysteine that is a portion of the active center of GSHperoxidase (Px) (Terovaet al., 2018). Therefore, dietary supplementation of Se is essential to improve Sedependent antioxidant enzymes. These enzymes can help in decreasing the concentration of lipid peroxides and hydrogen peroxide. Dietary Che-SeNP enhanced oxidative stability and antioxidant ability in broilers (Caiet al., 2012). Mohamed et al. (2016) reported a positive effect on plasma total antioxidant capacity of birds fed a diet containing Che-SeNP. Aparna and Karunakaran (2016) detected an increase in glutathione peroxidase and SOD cellular activity in birds fed Che-SeNP compared to the control group. ElDeep et al. (2016) displayed that Che-SeNP enhanced the activities of SOD and GSH-Px and reduced MDA content in the liver of broilers. The improvement of antioxidant status in quails fed Che-SeNP in the current study may be attributed to that (1) Che-SeNP had high antioxidant activity because it has an augmented ability to trap free radicals with better antioxidant influence, (2) Che-SeNP can act as a chemo preventive agent when administered at a smaller particle size, (3) Se plays a key role as an antioxidant that could protect intestinal mucosa against pathogens and oxidative damage, and (4) Se has immunomodulation properties (Torres et al., 2012).

Nanominerals such as Che-SeNP can increase immune parameters and disease resistance (El-Deep et al., 2016). In the current study, we presented a potential approach to the application of Che-SeNP to improve the immunity of quails. These findings can be due to the higher absorption of selenium nanoparticles. The present data are in harmony with the study of Cai et al. (2012), who stated that dietary Che-SeNP supplementation improved humoral immunity by increasing the levels of IgG and IgM of broiler chicks. Dietary Che-SeNP supplementation showed immunostimulatory impacts in broiler chicks (Kadhim et al., 2018). The improvement in serum immunoglobulins levels may be attributed to the important biological role of Che-SeNP in increasing T helper cells and enhancing the secretion of cytokines (Shabani et al., 2019). Additionally, Se plays a key role in the production of GSH-Px. Selenium inhibits arachidonic acid peroxidation and protects cells and tissues of the immune system from damage caused by free radicals. Therefore, it can be said that Che-SeNP boosts the immunity and antioxidant metabolites of birds (Kadhim et al., 2018). 
The regulation of microbiota in the gut can be achieved through dietary supplements that can encourage the beneficial bacteria growth or can selectively suppress the activity of pathogenic bacteria. Trace elements and natural agents as feed additives may affect the diversity of gut microbiota (Reda et al., 2020). The present study found that supplementation of Che-SeNP in quail diets declined harmful bacteria and increased useful bacteria. Se is one of the important elements that can help microbiota to complete its action within the gut (Yoon et al., 2007). Se supplementation augmented the population of caecum such as Bifidobacterium spp. and Lactobacillus spp. compared to the basal diet (Yoon et al., 2007). Therefore, using Che-SeNP is one of the recommendations for reducing the population of harmful gut bacteria due to its inhibitory effect against many pathogenic bacteria.

\section{Conclusion}

The findings of the current study demonstrated that dietary supplementation with Che-SeNP could improve the performance of growing quails; the best level was $0.4 \mathrm{~g}$ Che-SeNP/kg feed. Additionally, the physiological and health statuses were enhanced with the dietary addition of Che-SeNP that improved the lipid profile, antioxidant indices, and immunity, as well as decreased the intestinal pathogens of growing quails.

\section{Declarations}

\section{DECLARATION OF INTEREST}

No potential conflicts of interest declared.

\section{ACKNOWLEDGEMENTS}

The authors extend thanks to their respected institutes and universities.

\section{References}

1. Ahmadi M, Ahmadian A, Seidavi AR. Effect of different levels of nano-selenium on performance, blood parameters, immunity and carcass characteristics of broiler chickens. Poultry Science Journal. 2018;6:99-108.

2. Aparna N, Karunakaran R. Effect of selenium nanoparticles supplementation on oxidation resistance of broiler chicken. Indian Journal of Science Technology. 2016;9:1-5.

3. Bakhshalinejad R, Hassanabadi A, Swick RA. Dietary sources and levels of selenium supplements affect growth performance, carcass yield, meat quality and tissue selenium deposition in broilers. Animal Nutrition. 2019;5:256-63.

4. Bhunchu S, Muangnoi C, Rojsitthisak P. Curcumin diethyl disuccinate encapsulated in chitosan/alginate nanoparticles for improvement of its in vitro cytotoxicity against MDA-MB-231 
human breast cancer cells. Die Pharmazie-An International Journal of Pharmaceutical Sciences. 2016;71(12):691-700.

5. Boostani A, Sadeghi AA, Mousavi SN, Chamani M, Kashan N. The effects of organic, inorganic, and nano-selenium on blood attributes in broiler chickens exposed to oxidative stress. Acta Scientiae Veterinariae. 2015;43:1-6.

6. Cai SJ, Wu CX, Gong LM, Song T, Wu H, Zhang LY. Effects of nano-selenium on performance, meat quality, immune function, oxidation resistance, and tissue selenium content in broilers. Poult Sci. 2012;91:2532-9.

7. Chen G, Wu J, Li C. The effect of different selenium levels on production performance and biochemical parameters of broilers. Italian Journal of Animal Science. 2013;12:e79.

8. El-Deep MH, ljiri D, Ebeid TA, Ohtsuka A. Effects of dietary nano-selenium supplementation on growth performance, antioxidative status, and immunity in broiler chickens under thermoneutral and high ambient temperature conditions. The Journal of Poultry Science. 2016;53:274-83.

9. Elsaid AE. 2015. Influence of in-ovo injection with nanoparticles selenium (SeNPs) on growth performance, carcass yield and immune status of broiler chicks PhD Thesis. Damietta University, Faculty of Agriculture. Damietta (Egypt).

10. Estevam EC, Griffin S, Nasim MJ, Denezhkin P, Schneider R, Lilischkis R. Natural selenium particles from Staphylococcus carnosus: Hazards or particles with particular promise? J Hazard Mater. 2017;324:22-30.

11. Fesharaki PJ, Nazari P, Shakibaie M, Rezaie S, Banoee M, Abdollahi M, Shahverdi AR. Biosynthesis of Selenium Nanoparticles using Klebsiella pneumonia and their recovery by a simple sterilization process. Brazilian Journal of Microbiology. 2010;41:461-6.

12. Hariharan $\mathrm{H}$, Al-Harbi NA, Karuppiah P, Rajaram S. Microbial synthesis of selenium nanocomposite using saccharomyces cerevisiae and its antimicrobial activity. Chalcogen Letters. 2012;9(12):50915.

13. Huang $X$, Chen $X$, Chen $Q, Y u$ Q, Sun D, Liu J. Investigation of functional selenium nanoparticles as potent antimicrobial agents against superbugs. Acta Biomater. 2016;30:397-407.

14. Ibrahim NS, Sabic EM, Wakwak MM, EI-Wardany IE, El-Homosany YM, Mohammad NED. In-ovo and dietary supplementation of selenium nano-particles influence physiological responses, immunological status and performance of broiler chicks. Journal of Animal Feed Sciences. 2020;29:46-58.

15. Ismail FSA, Mostafa MY, Azzam MMM, Gorgy MAL. Effect of some sources of antioxiadnts on the productive and reproductive performance of turkey hens. Journal of Animal Poultry Production. 2016;7:393-401.

16. Joshua PP, Valli C, Balakrishnan V. Effect of in ovo supplementation of nano forms of zinc, copper, and selenium on post-hatch performance of broiler chicken. Veterinary world. 2016;9:287.

17. Khazraie S, Ghazanfarpoor R. Effect of nano-selenium particles and sodium selenite on performance, glutathione peroxidase and superoxide dismutase of quail under heat stress. International Journal of 
Life Sciences. 2015;5:875-82.

18. Kheradmand E, Rafii F, Yazdi MH, Sepahi AA, Shahverdi AR, Oveisi MR. The antimicrobial effects of selenium nanoparticle-enriched probiotics and their fermented broth against Candida albicans. DARU Journal of Pharmaceutical Sciences. 2014;22:1-6.

19. Khiralla GM, El-deeb BA. Antimicrobial and antibiofilm effects of selenium nanoparticles on some foodborne pathogens. LWT Food ScienceTechnology. 2015;63:1001-7.

20. Klopotek A, Hirche F, Eder K. PPARy ligand troglitazone lowers cholesterol synthesis in HepG2 and Caco-2 cells via a reduced concentration of nuclear SREBP-2. Experimental Biology Medicine. 2006;231:1365-72.

21. Mohamed HS, Rizk YS, Elslamony AE, Soliman AA, Ebrahim AF. Study the relationship between selenium and heat shock proteins under heat stress for local sinai chickens strain. Egyptian Poultry Science Journal. 2016;36:337-54.

22. Phull A, Abbas Q, Ali A, Raza H, Ja S, Zia M. Antioxidant, cytotoxic and antimicrobial activities of green synthesized silver nanoparticles from crude extract of Bergenia ciliata. Future Journal of Pharmaceutical Sciences. 2016;2:31-6.

23. Pilarczyk B, Jankowiak D, Tomza-Marciniak A, Pilarczyk R, Sablik P, Drozd R, Skólmowska M. Selenium concentration and glutathione peroxidase (GSH-Px) activity in serum of cows at different stages of lactation. Biol Trace Elem Res. 2012;147:91-6.

24. Reda FM, El-Saadony MT, Elnesr SS, Alagawany M, Tufarelli V. 2020. Effect of dietary supplementation of biological curcumin nanoparticles on growth and carcass traits, antioxidant status, immunity and caecal microbiota of japanese quails. Animals 2020, 10, 754.

25. Rizk YS, Ibrahim AF, Mansour MK, Mohamed HS, El-Slamony AE, Soliman AAM. Effect of dietary source of selenium on productive and reproductive performance of Sinai laying hens under heat stress conditions. Egyptian Poultry Science Journal. 2017;37:461-89.

26. Selim NA, Radwan NL, Youssef SF, Eldin TS, Elwafa SA. Effect of inclusion inorganic, organic or nano selenium forms in broiler diets on: 1-growth performance, carcass and meat characteristics. International Journal of Poultry Science. 2015;14:135.

27. Shabani R, Fakhraei J, Yarahmadi HM, Seidavi A. Effect of different sources of selenium on performance and characteristics of immune system of broiler chickens. Revista Brasileira de Zootecnia. 2019;48:e20180256.

28. Shakibaie M, Forootanfar H, Golkari Y, Mohammadi- Khorsand T, Shakibaie MR. Anti-biofilm activity of biogenic selenium nanoparticles and selenium dioxide against clinical isolates of Staphylococcus aureus, Pseudomonas aeruginosa and Proteus mirabilis. J Trace Elem Med Biol. 2015;29:235-41.

29. Shirsat S, Kadam A, Naushad M, Mane RS. Selenium nanostructures: microbial synthesis and applications. Rsc Advances. 2015;5:92799-811.

30. Tayeb I, Qader G. Effect of feed supplementation of selenium and vitamin E on production performance and some hematological parameters of broiler. KSU Journal of Natural Sciences. 2012;15:46-56. 
31. Terova G, Rimoldi S, Izquierdo M, Pirrone C, Ghrab W, Bernardini G. Nano-delivery of trace minerals for marine fish larvae: influence on skeletal ossification, and the expression of genes involved in intestinal transport of minerals, osteoblast differentiation, and oxidative stress response. Fish Physiol Biochem. 2018;44:1375-91.

32. Torres SK, Campos VL, León CG, Rodríguez-Llamazares SM, Rojas SM, Gonzalez M, Mondaca MA. Biosynthesis of selenium nanoparticles by Pantoea agglomerans and their antioxidant activity. $J$ Nanopart Res. 2012;14:1236.

33. Yoon I, Werner TM, Butler JM. Effect of source and concentration of selenium on growth performance and selenium retention in broiler chickens. Poult Sci. 2007;86:727-30.

34. Zhang J, Wang X, Xu T. Elemental selenium at nano size (Nano-Se) as a potential chemopreventive agent with reduced risk of selenium toxicity: comparison with se-methylselenocysteine in mice. Toxicol Sci. 2008;101:22-31.

35. Zhou $X$, Wang $Y$. Influence of dietary nano elemental selenium on growth performance, tissue selenium distribution, meat quality, and glutathione peroxidase activity in Guangxi Yellow chicken. Poult Sci. 2011;90:680-6. 